\title{
Impact of Pass/Fail USMLE Step 1 Scoring on the Internal Medicine Residency Application Process: a Program Director Survey
}

J Gen Intern Med 36(8):2509-10

DOI: $10.1007 / \mathrm{s} 11606-020-05984-y$

(c) Society of General Internal Medicine 2020

\section{INTRODUCTION}

In February of 2020, it was announced that U.S. Medical Licensing Examination (USMLE) Step 1 results would be reported as binary (pass/fail) as early as 2022 . A major motivation for this change was to reduce emphasis on USMLE scores in the transition between undergraduate and graduate medical education. ${ }^{1}$ Numeric Step 1 scores play a major role in the internal medicine (IM) residency application process in part because there are far more applicants than available positions. $^{2}$ In 2018, 95\% of IM program directors (PDs) used Step 1 scores to determine who to interview for their residency programs. $^{3}$ A recent national survey demonstrated negative PD perceptions of binary Step 1 scoring across many specialties. ${ }^{4}$ The perspective of internal medicine PDs, however, remains uncharacterized. This study aims to determine IM PDs' perspectives regarding the effect of pass/fail USMLE Step 1 scoring on the residency application process.

\section{METHODS}

After survey validation and institutional review board exemption, we invited PDs from Accreditation Council for Graduate Medical Education (ACGME)-accredited residency programs in 30 specialties, including 502 IM PDs, to voluntarily participate in an anonymous electronic survey. ${ }^{4}$

\section{RESULTS}

Two hundred six responses were obtained (response rate $=$ 41.0\%). The mean PD age was $52 \pm 9.8$ years. $65.0 \%$ were male. The mean tenure as PD was $7.4 \pm 6.4$ years. Only $11.7 \%$ of PDs agreed that the scoring change was a good idea, and few (18.9\%) agreed it would improve medical student wellbeing. $74.8 \%$ anticipated the change would make applicant screening more arduous. The majority of PDs (88.4\%) reported that binary Step 1 scoring would increase emphasis

Received May 6, 2020

Accepted June 11, 2020

Published online June 30, 2020 on USMLE Step 2 Clinical Knowledge (CK), and $83.6 \%$ would require Step $2 \mathrm{CK}$ scores at the time of application. Meanwhile, $57.3 \%$ of PDs reported that medical school reputation would become more important. Finally, only $3.4 \%$ of PDs believed Step 2 CK should be changed to pass/fail (Table 1).

\section{DISCUSSION}

The purpose of this study was to characterize the implications of binary Step 1 scoring within internal medicine. While a primary goal of this change was to decrease emphasis on standardized test scores, our results suggest that Step 2 CK will likely replace Step 1 in the application process. ${ }^{1}$ Another objective of the scoring change was to improve medical student wellness given the significant stress associated with USMLE exams. ${ }^{5}$ Unfortunately, only $18.9 \%$ of IM PDs believe medical student well-being will improve. This is unsurprising, as overreliance on high-stakes exams causes anxiety, and shifting the emphasis of test-performance to Step 2 CK leaves the root of the problem unaddressed.

In contrast to many other specialties, IM PDs already prioritize Step 2 CK performance. In fact, 90\% of programs use Step 2 CK to screen applicants for interviews, and most PDs give Step 2 CK similar weight as Step 1. ${ }^{3}$ Previously, strong performances on Step 2 CK could help remediate low Step 1 scores. However, without a numeric Step 1 score, there will be even more pressure to perform well on Step 2 CK. Additionally, students who fail Step 1 on the first attempt can no longer demonstrate significant numeric improvement on the repeat exam.

Worryingly, $57.3 \%$ of PDs report medical school reputation will be of greater importance in the application process. In 2019, IMGs and osteopathic students filled over half (55.8\%) of PGY-1 positions in IM. ${ }^{2}$ Without Step 1 scores to allow for standardized comparison between these students and their US allopathic counterparts, IMGs and osteopathic applicants may be at a disadvantage in the match.

In summary, most IM PDs do not support binary Step 1 scoring. Few PDs believe the change will improve medical student well-being, and most think it will make the residency selection process more arduous. This change will increase emphasis on USMLE Step 2 CK and disadvantage certain groups of medical students. Though the change to pass/fail scoring is well-intentioned, until additional reforms are 
Table 1 Internal Medicine Program Director Perspectives of Scoring Step 1 Pass/Fail

\begin{tabular}{|c|c|c|c|}
\hline \multirow[t]{2}{*}{ Statement } & Disagree & Neutral & Agree \\
\hline & \multicolumn{3}{|c|}{ Percent $(99.9 \%$ confidence interval) } \\
\hline \multicolumn{4}{|l|}{ Changing the USMLE Step 1 to pass/fail: } \\
\hline Is a good idea & $73.2(63.0-83.4) *$ & $15.1(6.9-23.4)$ & $11.7(4.3-19.1)$ \\
\hline Will make it more difficult to objectively compare applicants & $8.7(2.3-15.1)$ & $10.1(3.2-17.1)$ & $81.2(72.2-90.1) *$ \\
\hline Will increase emphasis on Step $2 \mathrm{CK}$ scores in selecting applicants for my program & $4.8(0.0-9.7)$ & $6.8(1.0-12.5)$ & $88.4(81.1-95.7) *$ \\
\hline Will put international medical graduates at a disadvantage & $14.6(6.5-22.7)$ & $29.6(19.1-40.1)$ & $55.8(44.4-67.2) *$ \\
\hline Will decrease socioeconomic disparities in the application process & $45.4(33.9-56.8)$ & $42.9(31.5-54.3)$ & $11.7(4.3-19.4)$ \\
\hline Will decrease medical student knowledge of the basic sciences & $18.4(9.6-27.3)$ & $39.3(28.1-50.5)$ & $42.2(30.9-53.6)$ \\
\hline Will improve medical student well-being & $43.7(32.3-55.1)$ & $37.4(26.3-48.5)$ & $18.9(9.9-27.9)$ \\
\hline Will make applicant screening more arduous & $9.2(2.6-15.9)$ & $16.0(7.6-24.4)$ & $74.8(64.8-84.7) *$ \\
\hline \multicolumn{4}{|l|}{ As a result of changing USMLE Step 1 to pass/fail: } \\
\hline I will now require applicants to submit Step $2 \mathrm{CK}$ scores with ERAS & $3.6(0.0-8.4)$ & $12.7(4.2-21.3)$ & $83.6(74.2-93.1) *$ \\
\hline $\begin{array}{l}\text { Where an applicant goes to medical school will be more important in screening and } \\
\text { selection for my program }\end{array}$ & $17.6(8.7-26.5)$ & $25.1(15.0-35.2)$ & $57.3(45.7-68.8) *$ \\
\hline Step $2 \mathrm{CK}$ should also be changed to pass/fail & $88.8(81.6-96.1) *$ & $7.8(1.6-13.9)$ & $3.4(0.0-7.6)$ \\
\hline
\end{tabular}

*Indicates a statistically significant $(p<0.01)$ plurality of responses by non-overlapping $99.9 \%$ confidence intervals

implemented, it may negatively affect the IM residency application process for both applicants and programs.

Arrush Choudhary, $B A^{1}$

Alan T. Makhoul, BA ${ }^{1}$

Nishant Ganesh Kumar, $M D^{2}$

Brian C. Drolet, MD, FACS ${ }^{3}$

${ }^{1}$ School of Medicine, Vanderbilt University,

Nashville, TN, USA

${ }^{2}$ Section of Plastic Surgery, Department of Surgery,

University of Michigan Health System,

Ann Arbor, MI, USA

${ }^{3}$ Department of Plastic Surgery, Department of Biomedical Informatics, Center for Biomedical Ethics and Society, Vanderbilt University Medical Center,

Nashville, TN 37212, USA

Corresponding Author: Brian C. Drolet, MD, FACS; Department of Plastic Surgery, Department of Biomedical Informatics, Center for Biomedical Ethics and Society, Vanderbilt University Medical CenterNashville, TN 37212, USA (e-mail: brian.c.drolet@gmail.com).

\section{Compliance with Ethical Standards:}

Conflict of Interest: The authors have no relevant conflicts of interest to disclose related to this manuscript. Please see attached ICMJE form for further details.

\section{REFERENCES}

1. Summary Report and Preliminary Recommendations from the Invitational Conference on USMLE Scoring (InCUS), March 11-12, 2019. United States Medical Licensing Examination. Available at: https://www.usmle.org/ pdfs/incus/incus_summary_report.pdf. Accessed March 29, 2020.

2. Results and Data 2019 Main Residency Match. National Resident Matching Program. Available at: https://mk0nrmp3oyqui6wqfm.kinstacdn.com/ wp-content/uploads /2019/04 /NRMP-Results-and-Data-2019_ 04112019 final.pdf. Accessed April 2, 2020.

3. Results of the 2018 NRMP Program Director Survey. National Resident Matching Program Data Release and Research Committee. Available at: https://www.nrmp.org/wp-content/uploads/2018/07/NRMP-2018-Program-Director-Survey-for-WWW.pdf. Accessed March 28, 2020.

4. Makhoul AT, Pontell ME, Ganesh Kumar N, Drolet BC. Objective measures needed - program directors' perspectives on a Pass/Fail USMLE Step 1. N Engl J Med. 2020;382(25):2389-2392.

5. Prober CG, Kolars JC, First LR, Melnick DE. A Plea to Reassess the Role of United States Medical Licensing Examination Step 1 Scores in Residency Selection. Acad Med. 2016;91(1):12-5.

Publisher's Note: Springer Nature remains neutral with regard to jurisdictional claims in published maps and institutional affiliations. 